\title{
Rational in silico design of aptamers for organophosphates based on the example
}

\section{of paraoxon}

Daria A. Belinskaia ${ }^{a,}{ }^{*}$, Pavel V. Avdonin ${ }^{b}$, Piotr P. Avdonin ${ }^{b}$, Richard O. Jenkins ${ }^{c}$, Nikolay V. Goncharova,d

a Sechenov Institute of Evolutionary Physiology and Biochemistry, Russian Academy of

Sciences, pr. Torez 44, St. Petersburg 194223, Russia

boltzov Institute of Developmental Biology, Russian Academy of Sciences, 26

Vavilova str., Moscow 119334, Russia

'Leicester School of Allied Health Sciences, De Montfort University, The Gateway, Leicester LE1 9BH, UK

dResearch Institute of Hygiene, Occupational Pathology and Human Ecology, bld.93 p.o.Kuz'molovsky, Leningrad Region 188663, Russia

* Corresponding author,

Telephone: 0079215806919

E-mail: d_belinskaya@mail.ru 


\begin{abstract}
Poisoning by organophosphates (OPs) takes one of the leading places in the total number of exotoxicoses. Detoxication of OPs at the first stage of the poison entering the body could be achieved with the help of DNA- or RNA-aptamers, which are able to bind poisons in the bloodstream. The aim of the research was to develop an approach to rational in silico design of aptamers for OPs based on the example of paraoxon. From the published sequence of an aptamer binding organophosphorus pesticides, its threedimensional model has been constructed. The most probable binding site for paraoxon was determined by molecular docking and molecular dynamics (MD) methods. Then the nucleotides of the binding site were mutated consequently and the values of free binding energy have been calculated using MD trajectories and MM-PBSA approach. On the basis of the energy values, two sequences that bind paraoxon most efficiently have been selected. The value of free binding energy of paraoxon with peripheral anionic site of acetylcholinesterase (AChE) has been calculated as well. It has been revealed that the aptamers found bind paraoxon more effectively than $\mathrm{AChE}$. The peculiarities of paraoxon interaction with the aptamers nucleotides have been analyzed. The possibility of improving in silico approach for aptamer selection is discussed.
\end{abstract}

\title{
Keywords:
}

Aptamer; Molecular docking; Molecular dynamics; Rational design; Organophosphates 


\section{Introduction}

Organophosphates (OPs) are widely used in agriculture and industry as pesticides, plasticizers, components of medicines, polymeric materials, and can be used as chemical weapon as well (Costa, 2006; Pope et al., 2005; Rousseau et al., 2000; Pitschmann, 2014). That is why poisoning by OPs has been taking one of the leading places in the total number of exotoxicoses (Dharmani and Jaga, 2005; Peter et al., 2010). Since the main mechanism of OPs is inhibition of acetylcholinesterase (AChE), the existing approaches of therapy for acute poisoning are reduced to eliminating the effects of OPs on AChE (Kaur et al., 2014; King and Aaron, 2015). Such approaches do not always prevent the irreversible effects of poisoning. Even in the case of survival, injured patients may experience a delayed pathology: so-called "intermediate syndrome"; central peripheral distal sensory-motor axonopathy; symptoms of vegetative changes in cardiovascular system; "microorganism disorders" of the central nervous system of unclear etiology; etc. (Lotti et al., 1993; Ray, 1998; Radilov et al., 2009; Goncharov et al., 2017). The effectiveness of the existing antidotal, symptomatic and preventive therapy can be significantly increased by increasing the detoxification of OPs at the first stage of entry of the poison into the body - in the bloodstream, which will reduce the toxic effect of OPs on the $\mathrm{AChE}$ of neuromuscular and neuronal synapses. High-affinity binding of OPs in the bloodstream could be achieved using DNA or RNA aptamers, which are short strands of oligonucleotides (Ku et al., 2015). Aptamers per se are nucleotide analogues of antibodies, but the process of synthesis of aptamers is much simpler and cheaper (Conrad et al., 1996; Kulbachinskiy, 2007). Aptamers are not attacked by the immune system and are non-toxic (Bouchard et al., 2010). All these properties make aptamers the ideal candidates for therapeutic purposes. 
At present, the main method of selection of aptamers is the method of systematic evolution of ligands by exponential enrichment (SELEX). The process is based on the stepwise selection and amplification of oligonucleotide sequences having the maximum affinity for a target molecule (Hüttenhofer and Vogel 2006; Stoltenburg et al., 2007; Wang et al., 2012). A huge number of aptamers capable of binding various inorganic and organic molecules, proteins and whole cells have been selected so far $(\mathrm{Ku}$ et al., 2015). However, relatively few studies have been devoted to identification of nucleotide biosensors for OPs; only the aptamers for four organophosphorus pesticides (forate, profenophos, isocarbophos, omethoate) and for the insecticide malathion have been found (Wang et al., 2012; Zhang et al., 2014; Williams et al., 2014). The main difficulty in selection of aptamers for OPs is due to the fact that molecules of OPs are structurally similar, which makes it difficult to choose an aptamer that not only binds a molecule of organophosphate but also binds it with high specificity, which could be important in the case of a contact with a particular insecticide used in agriculture or with a nerve agent to be expected with high probability in the case of a terrorist attack. There are difficulties in the methodology of selection, due to the fact that molecules of aptamers are several times larger than molecules of OPs. This can lead to high level interference during the process of detection. Therefore, only sensitive methods are able to detect interaction of aptamers with molecules of OPs, such as plasmon resonance, isothermal titration calorimetry and capillary electrophoresis. However, each of the sensitive methods has its own set of restrictions and cannot always be applied.

The methods of molecular modeling could replace SELEX and avoid the difficulties of this approach. For example, Ashrafuzzaman et al. (2013) attempted to construct a DNA aptamer for phosphatidylserine using in silico tools. They used a method similar to the 
SELEX approach: for each of the four possible nucleotides, the probability of its interaction with phosphatidylserine molecule was calculated, the most probable pairing was chosen, then again the probabilities of interaction of the ligand-nucleotide pair obtained at the previous stage with four nucleotides were calculated, etc. Shcherbinin et al. (2015) applied an in silico approach to find an aptamer for cytochrome P450. Having constructed 64 possible sequences of three nucleotides, the authors performed molecular docking of these sequences into the protein binding site and selected the most effectively binding triplets. However, the approaches described have a restriction: they can only find the binding fragment of the aptamer, and it is not possible to determine the structure of the whole aptamer. In the work (Shcherbinin et al., 2015) the non-binding part of the aptamers for cytochrome P450 were constructed based on the structure of the aptamer for thrombin determined earlier by SELEX. The combination of experimental and modeling methods, e.g. the improvement of experimentally obtained aptamers by in silico tools, could be a solution to the problem.

Such combination has been successfully applied by Hsieh et al. (2017) to determine the aptamers for prostatic specific antigen (PSA). Having taken five known aptamers for PSA as the basis, the authors applied the so-called genetic algorithm: "crossing-over" (exchange of nucleotides between sequences) and mutations (replacement of nucleotides in the same sequence). As a result, the sequences of the "next generation" were created, which bind PSA more efficiently and selectively than the original aptamers obtained by the SELEX method.

In the present work, we have applied for the first time the rational in silico design of the aptamer for OPs based on known experimental data on the example of paraoxon. According to the published sequence of an aptamer binding organophosphorus 
pesticides (Wang et al., 2012; Zhang et al., 2014), we have constructed a threedimensional model of the aptamer, determined the most probable binding site, and by mutating gradually the nucleic acids of the site, found the sequences binding the paraoxon molecule with the highest affinity.

\section{Materials and methods}

\subsection{Building of 3D-structure of aptamer}

The tertiary structure of the initial aptamer, consisting of 35 nucleotides, was constructed using an approach similar to one described by Bruno et al. (2008). The aptamer was constructed step by step on the basis of segments of its known secondary structure (Zhang et al., 2014) using HyperChem software (Froimowitz, 1993):

\section{Duplex 5'-A ${ }^{1} \mathrm{GCT}-3^{\prime}: 5^{\prime}-\mathrm{A}^{32} \mathrm{GCT}-3^{\prime}$}

$$
\text { Pin } 5^{\prime}-\mathrm{T}^{5} \mathrm{G}-3^{\prime}
$$

Duplex 5'-C ${ }^{7}$ TG-3' : 5' $-C^{29}$ AG-3'

$$
\text { Pin } 5^{\prime}-C^{10} A-3^{\prime}: 5^{\prime}-C^{27} A-3^{\prime}
$$

Duplex 5'-G ${ }^{12}$ CGAT-3' : 5'-A ${ }^{22}$ TCGC

\section{Pin $5^{\prime}-\mathrm{T}^{17} \mathrm{CTTG}-3^{\prime}$}

After construction of each segment, the structure was optimized by energy minimization using the conjugate gradient method (Fletcher, 1964). Then the next segment was added to the constructed fragment of the aptamer, etc. The final structure was optimized by 10 ns molecular dynamic simulation and then optimized by energy minimization using the 
conjugate gradient method. The details of all MD simulations are described in section 2.4 .

2.2 Molecular docking of paraoxon into aptamer binding sites and peripheral anionic site of acetylcholinesterase

Molecular docking of paraoxon into aptamer binding sites and peripheral anionic site (PAS) of acetylcholinesterase (AChE) was performed using Autodock 4.2 software (Morris et al., 1998). The structure of the aptamer obtained in the previous step was used as its 3D-model. The phosphate groups of the nucleotides of the aptamers were assumed to be single deprotonated, while the 5'-end nucleotide was assumed to be double protonated, giving the total charge of the aptamers equal to -36 . The structure 4m0e (Cheung et al., 2013) of human AChE from a PDB database (Berman et al., 2000) was used as a $3 \mathrm{D}$-model of $\mathrm{AChE}$. The total charge of $\mathrm{AChE}$ is -10 . The water molecules were deleted from the structure, and the missing hydrogen atoms were added using Gromacs 5.0.4 (Berendsen et al., 1995) software package. In the studied binding sites, an approximation grid of size 60 nodes in $\mathrm{x}-, \mathrm{y}-$, and $\mathrm{z}$-directions and with a step of $0.0375 \mathrm{~nm}$ was constructed. Estimated energy of binding $(\Delta G)$ was used to calculate the optimum conformations, with dissociation constant $\left(K_{\mathrm{d}}\right)$ calculated using this value and the formula (Morris et al., 1998). The Lamarckian genetic algorithm for searching the optimal conformations was used (Morris et al., 1998). The search procedure was repeated 50 times for each protein-ligand pair, which resulted in 50 optimal conformations. For each of the binding sites, the conformation with the minimal $K_{\mathrm{d}}$ was selected as the final one and used as the starting structure for further molecular dynamics simulation. 


\subsection{Mutation of nucleotides of the aptamer}

Mutation of nucleotides in the three-dimensional structure of the aptamer was performed using Discovery Studio Visualizer software (BIOVIA, 2016). The tool

"Build and Edit Nucleic Acid" was used, which allows one to automatically build or change a nucleotide according to the standard topology, then to save a new structure in pdb-format.

2.4. Molecular dynamics simulation of free aptamer, paraoxon-aptamer and paraoxonAChE complexes

Conformational changes of paraoxon-aptamer and paraoxon-AChE complexes were calculated by the MD method using the Gromacs 5.0.4 (Berendsen et al., 1995) software package and CHARMM force field (Zhu et al., 2012). The topology file for paraoxon is available in the Supplementary material (paraoxon-charmm.docx). The parameters for all the bonds, angles and dihedrals of paraoxon are already included in the CHARMM force field; 22 files of the force field parameters are available at http://mackerell.umaryland.edu/download.php?filename=CHARMM ff params files/c harmm36-jul2017.ff.tgz. The complexes obtained by molecular docking were placed virtually into a cubic periodic box filled with water molecules. The TIP3P model was used to describe water molecules (Jorgensen et al., 1983). To neutralize a system, 36 or 10 sodium ions were added to aptamers-paraoxon and $\mathrm{AChE}$ complexes respectively. Simulations were performed at a temperature of $300 \mathrm{~K}$ and a pressure of 1 bar. Temperature and pressure were kept constant using a V-rescale thermostat (Bussi et al., 2007) and a Berendsen barostat (Berendsen, 1984), with coupling constants of $0.1 \mathrm{ps}$ and $1.0 \mathrm{ps}$, respectively. Long-range electrostatic interactions were treated by the 
particle-mesh Ewald method (Darden, 1993). Lennard-Jones interactions were calculated with a cut off of $1.0 \mathrm{~nm}$. The LINCS algorithm was used to constrain bonds length. Before running the MD simulations, all the structures were minimized by steepest descent energy minimization (Jensen, 2010) and equilibrated with 100 ps MD simulation. $10 \mathrm{~ns}$ MD simulation with the time step 0.002 ps was performed to optimize the three-dimensional structure of the aptamer. 2 ns MD simulation with the time step 0.0005 ps was performed to check stability of paraoxon-aptamer complexes. $10 \mathrm{~ns}$ MD simulation with the time step $0.002 \mathrm{ps}$ was performed to simulate conformational changes of the complexes of paraoxon with $\mathrm{AChE}$ and of the complexes of paraoxon with the chosen binding site (site-1) of the aptamers.

\subsection{Calculation offree binding energy of paraoxon-aptamer and paraoxon-AChE} complexes.

Free binding energies of the obtained complexes were calculated using the MM-PBSA method (Genheden and Ryde, 2015) with the help of g_mmpbsa application (Kumari et al., 2014) integrated into the Gromacs software package. The MM-PBSA method enables estimating of energy of receptor-ligand complex formation on the base of MD trajectories. Free binding energy $\Delta G_{\text {bind }}$ is calculated by the formula:

$$
\Delta G_{\text {bind }}=G_{\mathrm{RL}}-\left(G_{\mathrm{R}}+G_{\mathrm{L}}\right)(\text { eq. } 1),
$$

where $G$ is free energy, the index $\mathrm{R}$ refers to receptor, $\mathrm{L}$ - to ligand, $\mathrm{RL}$ - to receptorligand complex. Free energy of a molecule or a complex is calculated by the formula (2):

$$
G_{\mathrm{X}}=E_{\mathrm{MM}}+G_{\text {solv }}-T S_{\mathrm{MM}} \text { (eq. 2), }
$$


where the index $\mathrm{X}$ refers to a receptor, a ligand or their complex, $E_{\mathrm{MM}}$ is potential energy of a molecule or a complex in vacuum calculated by molecular mechanics method, $G_{\text {solv }}$ is free energy of solvation, $T S$ is the entropy component of free energy of a system in vacuum, $T$ and $S$ are temperature and entropy respectively.

Free energy of solvation is calculated by the formula:

$$
G_{\text {solv }}=G_{\text {polar }}+G_{\text {non-polar }}(\text { eq. } 3)
$$

where $G_{\text {polar }}$ and $G_{\text {non-polar }}$ are the contributions of polar and nonpolar interactions, respectively, to free energy of solvation. $E_{\mathrm{MM}}$ is calculated as the sum of covalent, van der Waals and electrostatic interactions.

Previous studies have showed that entropy value fluctuates greatly during MD simulation. It was shown that the calculation of the enthalpy component correlates better with the experimental data than the calculation of the total free energy (Kumari et al., 2014). Therefore, in our work we calculated the enthalpy component of free energy only.

In the work presented, we calculated the values of free energies every 1 ps during MD simulation. Thus, the result of every g_mmpbsa run was 10000 values of free energy. The final value was calculated as the mean \pm standard deviation; g_mmpbsa uses $\mathrm{kJ} / \mathrm{mol}$ as energy units. The obtained energy values were converted to $\mathrm{kcal} / \mathrm{mol}$.

\section{Results}

\subsection{Building the 3D-structure of aptamer}


In the work of Zhang et al. (2014), several DNA aptamers for four organophosphorus pesticides (isocarbophos, profenophos, omethoate, and phorate) were obtained by the SELEX method. Among the compounds, the structures of profenophos and isocarbophos are the most similar to the structure of paraoxon. The aptamer SS24-S-35, which has the highest affinity for profenophos and isocarbophos, has the following primary sequence:

\section{5'-AGCTTGCTGC ${ }^{10}$ AGCGATTCTT ${ }^{20}$ GATCGCCACA $^{30}$ GAGCT-3'}

Based on the secondary structure of the aptamer (Zhang et al., 2014), we have constructed its three-dimensional model and optimized the resulting structure by $10 \mathrm{~ns}$ MD simulation in solution. The change of root-mean-square deviation (RMSD) of atomic positions in time has been calculated. The value of RMSD increases during the first 3 ns of the simulation then remains unchanged during the last $7 \mathrm{~ns}$, which indicates stability of the structure. The final conformation of the aptamer was optimized by energy minimization. The final model is shown in Fig. 1.

\subsection{Molecular docking of paraoxon into the possible binding centers of the aptamer.}

Visual analysis has revealed five nucleotides (C18, A22, C13, A11 and T5) that are located at concavities of the obtained 3D structure of the aptamer and could be used as the grid centers (grid centers 1-5) for the molecular docking procedure for searching the sites where paraoxon could bind. Molecular docking of paraoxon into these five possible centers has been revealed five possible binding modes of paraoxon on the aptamer surface. We have repeated the search for possible grid centers using the following approach. Using g_mmpbsa package, we calculated the contribution of the 
nucleotides to the value of nonpolar solvation energy. Again, the structure of free aptamer obtained in section 2.1 was used. The value of nonpolar solvation energy is assumed to depend linearly on the value of solvent accessible surface area (Kumari et al., 2014). Thus, the nucleotides giving the minimal contribution into the value of nonpolar salvation energy have the minimal solvent accessible surface area. It means that they are located in the depth of the aptamer and could also be used as the grid centers for molecular docking procedure. The calculation of nonpolar solvation energy showed that the following nucleotides contribute the least: $\mathrm{C} 18\left(\Delta G_{\text {nonpolar }}=-0.51 \mathrm{kcal} /\right.$ mol, coincides with grid center 1$) ; \mathrm{A} 22\left(\Delta G_{\text {nonpolar }}=-0.69 \mathrm{kcal} / \mathrm{mol}\right.$, coincides with grid center 2); $\mathrm{A} 32\left(\Delta G_{\text {nonpolar }}=-0.82 \mathrm{kcal} / \mathrm{mol}\right.$, located in $3 \AA$ from grid center 5$) ; \mathrm{C} 26$ $\left(\Delta G_{\text {nonpolar }}=-0.82 \mathrm{kcal} / \mathrm{mol}\right.$, located in $4.6 \AA$ from grid center 3$) ; \mathrm{C} 10\left(\Delta G_{\text {nonpolar }}=-0.95\right.$ $\mathrm{kcal} / \mathrm{mol}$, located in $2.6 \AA$ from grid center 4). We have limited ourselves to five nucleotides, since five grids with centers at these points completely cover the surface of the aptamer. Thus, we have picked three additional grid centers: A32 as grid center 6, $\mathrm{C} 26$ as grid center 7, and $\mathrm{C} 10$ as grid center 8 . Three additional molecular docking procedures have been performed; the found binding modes 6-8 coincide with binding modes 5, 3 and 4 respectively. The final eight positions of the paraoxon molecules in sites 1-5 obtained by molecular docking are shown in Fig. 2.

For the complexes obtained, the values of intermolecular energy (sum of energies of van der Waals and electrostatic interactions) and the energy of hydrogen bonds and desolvation were calculated. In addition to the enthalpy component, the Autodock algorithm estimates the change of system entropy based on the amount of "frozen" rotating ligand bonds. Based on the values of enthalpy and entropy components, the free binding energy is estimated. The nucleotides of the binding sites $1-5$, the calculated 
values of intermolecular energy and the estimated values of free binding energy of paraoxon with the sites are shown in Table 1.

According to the energy values given in Table 1, site-5 is the most effective for paraoxon binding. However, sites- 1 and -4 have greater conformational mobility due to the fact that they contain unpaired nucleotides. Thus, despite the obtained energy values, sites- 1 and -4 are the most probable sites of paraoxon binding. To verify this hypothesis, the stability of all the complexes obtained was verified by $2 \mathrm{~ns} \mathrm{MD}$ simulation. Among the five complexes, only that of paraoxon with site-1 remained stable throughout the entire simulation. For the other four complexes, the paraoxon molecule became detached from the aptamer and then remained in an unbound state. As we anticipated, nucleotides of site-1 have sufficient conformational mobility to adjust to the paraoxon molecule. Moreover, in the papers of Wang (2012) and Zhang (2014), the authors obtained not only aptamer SS24-S-35 but several aptamers for organophosphate pesticides, and all of them contain the same sequence: CTGCAGCGATTCTTGATCG, which probably forms the binding site for the pesticides. In the case of the aptamer SS24-S-35, only sites-1 and -2 contain this sequence in its entirety. This fact is in agreement with the result of MD simulation that showed site- 1 to be the most stable binding site for paraoxon.

The complex of paraoxon with site- 1 after 2 ns of MD simulation became the starting structure for further mutations of nucleotides of the binding site, in order to find the sequence that binds paraoxon as efficiently as possible. The initial coordinates of paraoxon were the same for all the mutants. Hereinafter, for convenience, we use the 
abbreviation PAC (paraoxon-aptamer-complex) for the initial paraoxon-aptamer complex, which is the complex of aptamer SS24-S-35 with paraoxon bound in site-1.

\subsection{Molecular dynamics simulation of the initial paraoxon-aptamer complex (PAC).}

Conformational changes of PAC over a longer time interval have been calculated by 10 ns MD simulation. The complex remained stable throughout the simulation, i.e. the paraoxon molecule stayed associated with site-1 of the aptamer (Fig. 3). The final conformation of PAC is shown in Fig. 4.

Visual analysis of the complex revealed that the paraoxon molecule is surrounded by nucleotides T17, C18, T19 and T20. The benzene ring of the ligand interacts with the aromatic ring of nucleotide T19, the phosphorus group of nucleotide T17, and the ethyl groups of nucleotides $\mathrm{C} 18$ and T20. Visual analysis of the aptamer nucleotides fluctuations over time shown that the side chains of nucleotides T17 and T19 do not change their conformation during the simulation and strongly interact with the paraoxon molecule, while the side chains of $\mathrm{C} 18$ and T20 change their positions relative to the paraoxon molecule periodically. In the final conformation, C18 is distant from the ligand molecule, while T20 is close to it.

On the basis of the trajectory obtained, the value of free binding energy of the complex has been calculated to be $-31.0 \pm 3.8 \mathrm{kcal} / \mathrm{mol}$. The contribution of each of the four nucleotides of site- 1 to the value of the binding energy has also been calculated; the results are shown in Table 2.

The quantitative analysis of the contribution of individual nucleotides to the binding energy value (Table 2) coincides with the qualitative visual analysis of the 
conformational changes described above. Nucleotides T20 and C18 contribute minimally to the interaction, while T19 and T17 contribute maximally. These data explain the result of MD, according to which paraoxon interacts with site-1 more weakly than with other possible binding sites (see section 3.2). According to the results of MD of paraoxon into the free aptamer, the "weak" nucleotides C18 and T20 are closest to paraoxon, and the enhancement of aptamer-paraoxon interaction occurs after conformational changes of the oligonucleotide. We therefore decided that it would be most rational to start our search for better sequences for binding paraoxon with replacement of the more "weak" nucleotides T20 and C18, and to check whether other nucleotides in these positions interact more strongly with paraoxon.

\subsection{Mutation of nucleotide at position 20.}

By virtual replacement of thymine in position 20 with adenine, cytosine or guanine, three new variants of the aptamer complex with paraoxon have been obtained: T20A, T20C and T20G. Conformational changes of T20A, T20C and T20G have been calculated by $10 \mathrm{~ns}$ molecular dynamics simulation. T20A and T20C remained stable, with paraoxon remaining bound to the aptamers during the entire simulation, T20G disintegrated after 6 ns (Fig. 3). The binding energies of the complexes have been calculated on the basis of the trajectories obtained, the results are shown in Table 3. According to the values obtained, none of the substitutions improved the affinity of the aptamer to paraoxon. The replacement of thymine with adenine was equivalent; the binding energy of T20A $(-30.7 \pm 3.9 \mathrm{kcal} / \mathrm{mol})$ was comparable to the value of PAC binding energy $(-31.0 \pm 3.8 \mathrm{kcal} / \mathrm{mol})$. The replacements of thymine with guanine or 
cytosine decreased the affinity. Thus, according to the data obtained, either thymine or adenine should be at position 20 .

\subsection{Mutation of nucleotide at position 18.}

As previously (section 3.4), three new variants of the aptamer complex with paraoxon were obtained by replacing C18 of PAC with adenine, guanine or thymine: C18A, C18G and C18T. Conformational changes of C18A, C18G and C18T have been calculated by $10 \mathrm{~ns}$ MD simulation. $\mathrm{C} 18 \mathrm{G}$ and $\mathrm{C} 18 \mathrm{~T}$ remained stable; paraoxon remained bound with the aptamers during the entire simulation, C18A disintegrated after $6 \mathrm{~ns}$ (Fig. 3). The binding energies of the complexes have been calculated on the basis of the trajectories obtained, the results are shown in Table 3. According to the values obtained, none of the substitutions improved the affinity of the aptamer to paraoxon. The replacement of cytosine with thymine was equivalent: the binding energy of $\mathrm{C} 18 \mathrm{~T}(-30.6 \pm 3.6 \mathrm{kcal} / \mathrm{mol})$ was comparable to the value to the value of PAC binding energy $(-31.0 \pm 3.8 \mathrm{kcal} / \mathrm{mol})$. The replacements of cytosine with adenine or guanine decreased the affinity. Thus, according to the data obtained, either cytosine or thymine should be at position 18 .

\subsection{Mutation of nucleotide at position 19.}

As previously (sections 3.4 and 3.5), three new variants of the aptamer complex with paraoxon were obtained by replacing T19 of PAC with adenine, cytosine or guanine: T19A, T19C and T19G. Conformational changes of T19A, T19C and T19G have been calculated by $10 \mathrm{~ns}$ MD simulation. All the complexes remained stable; paraoxon remained bound with the aptamers during the entire simulation (Fig. 3). The binding 
energies of the complexes have been calculated on the basis of the trajectories obtained, the results are shown in Table 3. According to the values obtained, none of the substitutions improved the affinity of the aptamer to paraoxon; all the replacements decreased the affinity. Thus, according to the data obtained, only thymine should be at position 19.

\subsection{Mutation of nucleotide at position 17.}

As previously (sections 3.4-3.6), three new variants of the aptamer complex with paraoxon were obtained by replacing $\mathrm{T} 17$ of PAC with adenine, cytosine or guanine: T17A, T17C and T17G. Conformational changes of T17A, T17C, and T17G have been calculated by $10 \mathrm{~ns}$ MD simulation. All the complexes remained stable; paraoxon remained bound with the aptamers during the entire simulation (Fig. 3). The binding energies of the complexes have been calculated on the basis of the trajectories obtained, the results are shown in Table 3. According to the values obtained, the replacements of thymine with adenine decreased the affinity. The replacement of thymine with guanine was equivalent; the binding energy of $\mathrm{T} 17 \mathrm{G}-31.3 \pm 3.7 \mathrm{kcal} / \mathrm{mol}$ ) was comparable to the value of PAC binding energy $(-31.0 \pm 3.8 \mathrm{kcal} / \mathrm{mol})$. The replacements of thymine with cytosine improved the affinity; the binding energy of T17C was equal to $-32.3 \pm 3.0$ $\mathrm{kcal} / \mathrm{mol}$. Thus, according to the data obtained, only cytosine should be at position 17 .

\subsection{Double and triple mutations}

At demonstrated in sections 3.5-3.7, we have revealed which single mutations improve binding of paraoxon with site-1. Based on the data obtained, we have checked two double and one triple mutations of the nucleotides of site-1: T17C-C18T, T17C-T20A 
and T17C-C18T-T20A. The free binding energies of the complexes have been calculated by $10 \mathrm{~ns}$ MD simulation. All the complexes remained stable; paraoxon remained bound with the aptamers during the entire simulation (Fig. 3). The values were equal to $-32.8 \pm 3.1 \mathrm{kcal} / \mathrm{mol},-30.6 \pm 3.4 \mathrm{kcal} / \mathrm{mol}$ and $-29.6 \pm 3.2 \mathrm{kcal} / \mathrm{mol}$ for T17C-C18T, T17C-T20A and T17C-C18T-T20A respectively. These data indicate that the double mutation T17C-C18T increases the effectiveness of paraoxon binding, whereas the mutations T17C-T20A and T17C-C18T-T20A do not.

Thus, according to our calculations, the following two aptamers bind paraoxon most effectively:

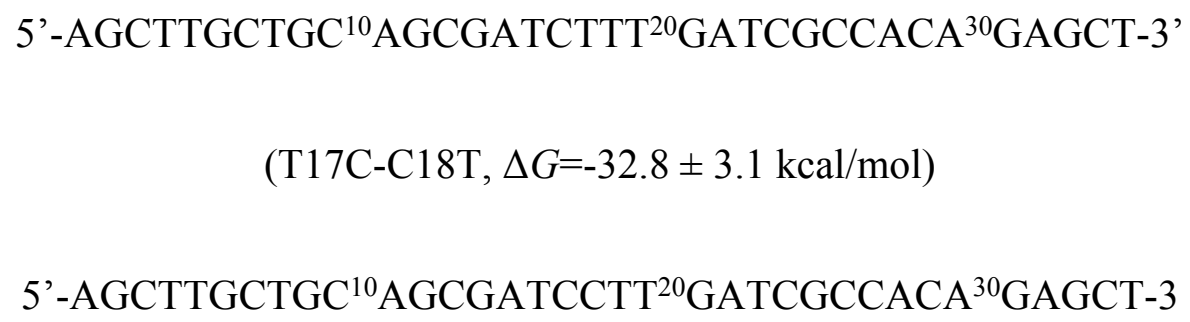

It is interesting to compare the values of binding effectiveness of our aptamers with in silico and in vivo data on binding properties of aptamers obtained by other researchers. According to the data given in Chang et al. (2014), the values of $K_{\mathrm{d}}$ of aptamers with small molecules vary in a very high range: from $200 \mathrm{pM}$ to $76 \mu \mathrm{M}$. As mentioned above, several aptamers for four organophosphate pesticides were obtained by the SELEX method (Wang et al., 2012). For two of these aptamers, the $K_{\mathrm{d}}$ values are given in the paper, with values varying from 0.83 to $2.5 \mu \mathrm{M}$. To understand what $K_{\mathrm{d}}$ we could expect from the vales of free binding energies obtained by us, we have turned to the work of Shcherbinin et al. (2015), where the binding characteristics of seven aptamers 
for cytochrome p450 were studied both by in silico and in vitro methods. The values of the enthalpy term of free binding energy for those seven aptamers, calculated by the MM-PBSA method, vary from -54.80 to $-14.72 \mathrm{kcal} / \mathrm{mol}$, and the experimentally obtained values of $K_{\mathrm{d}}$ vary from 0.14 to $1.3 \mu \mathrm{M}$. For three aptamers, the calculated values of enthalpy term were $-38.86,-37.76$ and $-36.26 \mathrm{kcal} / \mathrm{mol}$, which is comparable with the values obtained in our work (as mentioned above, we have calculated the enthalpy term of free biding energy only). For those three aptamers for cytochrome $\mathrm{p} 450$, the experimental $K_{\mathrm{d}}$ values were $0.4,0.5$ and $0.6 \mu \mathrm{M}$, respectively. Therefore, as a very rough approximation we can expect the $K_{\mathrm{d}}$ values of our aptamers for paraoxon to be around $1 \mu \mathrm{M}$.

\subsection{Binding of paraoxon with the peripheral anionic site of acetylcholinesterase.}

Synaptic acetylcholinesterase (AChE) is the main target of OPs. To estimate the ability of the aptamers found to compete with AChE for paraoxon binding, we have investigated interaction of paraoxon with the enzyme. It is known that the primary binding of ligands occurs in the peripheral anionic site (PAS) of AChE, which includes amino acids Tyr72 and Asp74 (numbering for human AChE). We have performed molecular docking of the paraoxon molecule into PAS. The complex obtained has then been used as the starting conformation for further $10 \mathrm{~ns}$ molecular dynamics simulation. The change in value of RMSD for the $\mathrm{C} \alpha$ atoms of $\mathrm{AChE}$ and for the paraoxon atoms relative to the starting conformation have been calculated. The value of RMSD for C $\alpha$ atoms of $\mathrm{AChE}$ increased up to 0.2 during the first $2 \mathrm{~ns}$ of the simulation, and then remained constant for the next $8 \mathrm{~ns}$. The RMSD value for paraoxon atoms increased up to 0.2 for the first $100 \mathrm{ps}$ of the simulation, and then remained constant. Visual analysis of the position of paraoxon in the PAS showed that the position of the ligand was stable 
throughout the entire simulation; the final conformation of the complex is shown in Fig. 5.

The atom of phosphoryl oxygen of paraoxon interacts with the NH group of Leu76, the benzene ring of the ligand is in the same plane with the aromatic group of Trp286, which indicates pi-pi interaction between them. One of the oxygen atoms of the nitro group of paraoxon forms a hydrogen bond with the backbone NH group of Asp74.

We have also calculated the free binding energy of AChE-paraoxon complex, which was $-26.0 \pm 2.6 \mathrm{kcal} / \mathrm{mol}$. Thus, according to the data obtained, the aptamers that we have found bind paraoxon more efficiently than $\mathrm{AChE}$.

The PAS of AChE is just the primary site for binding of AChE ligands. After binding of substrates and irreversible inhibitors with the PAS, conformational changes occur in the structure of $\mathrm{AChE}$ and ligands quickly penetrate further along the narrow active-site gorge towards the catalytic triad, where acetylation or irreversible phosphorylation of the catalytic serine occurs. Therefore, there are relatively few studies that distinguish ligand binding with the PAS and with the catalytic center. As for OPs, in the work of Radić et al. (1991) it was shown that $K_{\mathrm{d}}$ of the complex of PAS with haloxon (an organophosphate anthelmintic) equals $16 \mu \mathrm{M}$. Whilst this is ten-fold greater than our roughly estimated $K_{\mathrm{d}}$ for aptamer-paraoxon complexes, at least such a value does not contradict our data that the obtained aptamers binds paraoxon better that the PAS of AChE does.

Molecular docking procedure has revealed five possible binding sites of paraoxon on the aptamer surface. Furthermore, molecular dynamic simulation has shown that only site- 1 could bind paraoxon molecule effectively though, according to the results of 
docking, the binding in site-1 is the weakest (Table 1). We have proposed that such discrepancy could be due to greater conformational mobility of site-1, having five unpaired nucleotides. To this proposal, we have performed molecular docking of paraoxon into the improved conformational model of the aptamer. From the MD simulation of the initial aptamer with paraoxon, we have selected the conformation with the best binding energy $(\Delta G=-45.0 \mathrm{kcal} / \mathrm{mol})$, and then minimized this structure. The obtained structure $\left(\mathrm{Conf}_{\text {best }}\right)$ is shown in Fig. 6 in purple and grey. We have performed molecular docking of paraoxon into site- 1 of the aptamer in this conformation; the obtained geometry is shown in Fig. 6 in green. The orientation of the paraoxon molecule obtained by docking is close to the one in the complex selected from the MD trajectory. The value of intermolecular energy obtained by docking is $-5.79 \mathrm{kcal} / \mathrm{mol}$, and the estimated value of free binding energy is $-3.7 \mathrm{kcal} / \mathrm{mol}$, which is $1.4 \mathrm{kcal} / \mathrm{mol}$ lower than the value obtained by docking of paraoxon into site- 1 of the free relaxed aptamer, but still higher than the values for site-4 and 5 (Table 1).

This discrepancy between the results of docking and MM-PBSA can be explained by the fact that the empirical scoring function of Autodock estimate binding free energy by summing interaction terms derived from weighted structural parameters. The weights are obtained by fitting the scoring function to experimental binding constants of a training set of receptor-ligand complexes. Probably, such kind of parameterization is not ideal for DNA as a receptor. Also, Autodock does not take into account flexibilities of receptor-ligand complexes. The MM-PBSA method uses a force field based scoring function, and the main advantage of the g_mmpbsa module is that it takes several hundred snapshots of the free binding energy values from MD trajectory, which makes it possible to estimate the interaction properties with better approximation. But still, 
molecular docking can be used to determine the geometry of binding mode at a particular moment of time, which could be further evaluated by MD simulation.

It is interesting to compare the MM-PBSA approach and the linear interaction energy (LIE) method, which is an alternative method for free binding energy calculation (Åqvist et al. 1994). The LIE method postulates that the free energy of binding $\Delta G$ is linearly correlated to both van der Waals and electrostatic interactions between the ligand and the rest of the system. The LIE equation is known as:

$$
\Delta G=\alpha \Delta \mathrm{E}_{\mathrm{vdw}}+\beta \Delta \mathrm{E}_{\text {elec }}+\gamma \text { (eq. 4), }
$$

where $\Delta \mathrm{E}_{\mathrm{vdw}}$ and $\Delta \mathrm{E}_{\text {elec }}$ are the changes in van der Waals and electrostatic energy from the ligand free and bound state. The $\alpha, \beta$ and $\gamma$ are LIE empirical parameters, determined by comparing calculated and experimentally estimated binding affinities. MM-PBSA principles are described in section 2.5 Both approaches have their own advantages and disadvantages (Barril et al., 2001; Genheden, 2011; Genheden and Ryde, 2015; Homeyer et al.,2014; Hou et al., 2002; Mikulskis et al., 2012; Perdih et al., 2013). In the MM-PBSA approach, the solvation free energy is determined by a continuum solvent model and a term accounting for the non-polar contribution to solvation. Because of that, MM-PBSA needs only one MD simulation for the proteinligand complex, whereas two MD simulations for ligands in the bound and free states are needed for LIE. This is important because of the inverse correlation between calculation accuracy and required computing time. However, the calculations involving an estimation of solvation free energies at the macromolecular level can lead to large numerical errors. MM-PBSA is based on the general empirical parameters for PoissonBolzman and Surface Area calculations and one does not need to additionally calibrate 
the parameters. Conversely, the LIE method is highly system dependent and the optimization of the parameters can be required. When using MM-PBSA, one has to always decide whether to include the entropy or not. As it was mentioned above, the calculation of the enthalpy component correlates better with the experimental data than with the calculation of the total free energy. Hence, it seems reasonable to omit the entropy at a first stage for a ligand-receptor complex. Free energy changes produced by linear response models implicitly include conformational entropy effects through the linear response expressions, which relate potential energy differences to free-energy differences. It should be mentioned that the MM-PBSA method gives a large range of estimated affinities, while LIE gives results within a more reasonable range. Concluding the comparison of two approaches, it is possible to say that MM-PBSA is a reasonable choice for our calculations: (1) no additional parameterization for DNA as a receptor is needed and (2) the continuum solvent model reduced computation time greatly for more than twenty MD simulations performed in the paper.

The purpose of the study was not just to find the best aptamers for paraoxon but also to consider the possibility, based on currently available data, of developing the most rational approach to selection of aptamers to small molecules. Molecules of such a size as paraoxon and other OPs can interact with 3-5 nucleotides, which means that the number of possible combinations could vary from $4^{3}$ to $4^{5}$, which is $64-1024$ variants. In the present work, we have tried to optimize the search as much as possible. At the first step, we performed all possible single mutations of nucleotides of the original aptamer, and then, assuming that "minus" to "minus" does not give "plus" in our case, we studied the double and triple mutations that consist of "successful" single mutations only. In this approach we still had to test 16 combinations. Of course, we recognize that prescreening 
of effective aptamers by in silico methods could reduce the cost and speed up the procedure of bioscavengers selection. Nevertheless, it would be beneficial to optimize the prescreening process and to then select effective aptamers based on the chemical structure of a ligand. For example, a molecule of paraoxon contains three specific groups in its structure. Firstly, the phosphate group with a phosphorus atom having a huge positive charge and an oxygen atom having a large negative charge. Secondly, the nitro group with a positively charged nitrogen atom and two negatively charged oxygen atoms. We could expect that the oxygen atoms of the phosphate and nitro groups would form hydrogen bonds with NH-groups of nucleotides and "avoid" other oxygen atoms of an aptamer. According to such logic, the ideal neighboring groups would be adenosines. Thirdly, the paraoxon molecule contains a benzene ring, which would preferentially interact with aromatic groups of nucleotides via pi-pi interaction.

Further to the above theoretical expectations, we now consider the complex of paraoxon with the initial aptamer (PAC, Fig. 4). Although PAC is not the best aptamer, according to our data it still binds paraoxon better than AChE. It should be noticed that there are none of the expected interactions: neither pi-pi interaction between aromatic rings, nor hydrogen bonds between oxygen atoms of paraoxon and NH groups of nucleotides.

Fig. 7 shows the complexes of paraoxon with the most effective aptamers T17C-C18T (Fig. 7a) and T17C (Fig. 7b) that we have obtained.In the case of aptamer T17C, the benzene ring does form pi-pi interaction with nucleotide T19 (Fig.7b). However, for the complex of paraoxon with aptamer T17C-C18T, neither H-bonds nor pi-pi interaction are observed (Fig. 7a). 
Figures $4,7 \mathrm{a}$ and $7 \mathrm{~b}$ show the complexes of paraoxon with the initial aptamers, aptamer T17C and T17C-C18T after $10 \mathrm{~ns}$ of MD simulation. It should be noticed that the pose of the ligand differs even after only one mutation, which was probably due to the different charge distribution in nucleotides. Thus, thymine and cytosine differ from each other by one group: the ketone group $=\mathrm{O}$ in thymine is replaced by the $\mathrm{NH}_{2}$ group in cytosine. This affects the distribution of charges in the nucleotides. We used the CHARMM force field for the MD simulations. In this force field, the charge of atom O4 of thymine equals -0.45 , and the charge of atom N4 of the cytosine equal -0.75 . The charge of atom N1 equal -0.34 in thymine, and -0.13 in cytosine. The charge of atom N3 equal -0.46 in thymine, and -0.66 in cytosine. Since the position of paraoxon molecule in the complexes with the aptamers is less sterically restricted than if it were bound in some deep pocket of a protein like AChE, we suppose that difference in charge distribution can lead to such mobility of paraoxon in the binding site of the aptamers.

The conformation of a complex of a small molecule with an aptamer can be determined experimentally only by X-ray crystallography or nuclear magnetic resonance spectroscopy techniques, which are quite expensive procedures. Only a few dozen of such complexes have been published in the PDB database. Therefore, unfortunately, no one has tested experimentally how one nucleotide mutation affects the conformation of a ligand in aptamer binding sites. The work of Shcherbinin et al. (2015) could be used as an indirect confirmation that one mutation can significantly change the binding characteristics. The authors performed mutation of the binding moiety of the aptamers for cytochrome $\mathrm{p} 450$ (in this case the ligand is not a small molecule but a protein) and checked by in silico and in vitro methods how the mutation changed the binding properties of the aptamers. There were two aptamers in the testing set, differing only by 
one nucleotide (binding triplets CAT and CGT), and the values of dissociation constants for their complexes with cytochrome determined experimentally are 0.6 and $0.28 \mu \mathrm{M}$, respectively, which differ twice.

We have analyzed the contribution of electrostatic and van der Waals terms to interaction energy of paraoxon with aptamers T17C-C18T and T17C during the MD simulation period. The results are shown in Fig. 8(a-b). In the case of aptamer T17C$\mathrm{C} 18 \mathrm{~T}$, the value of electrostatic energy begins to increase after $3 \mathrm{~ns}$ of simulation from negative values to 0 , and van der Waals energy decreases (Fig. 8a). An analysis of the conformation of paraoxon in the binding site showed that in the time interval from 0 to $3 \mathrm{~ns}$ there is a pi-pi bond between paraoxon and nucleotide T20, then the bond breaks and the ligand transforms into a conformation corresponding to the final position (Fig. 7a). In the case of aptamer $\mathrm{T} 17 \mathrm{C}$, two main conformational states of its complex with paraoxon are observed during the simulation too. In the first one, the pi-pi bond is formed between paraoxon and the aromatic ring of nucleotide T19. This conformational state corresponds to stronger electrostatic interaction and weaker van der Waals interaction (periods $0-1$ and 6-10 ns of the simulation). In the second conformational state, there is no pi-pi interaction between the ligand and the aptamer (period 1-6 ns of the simulation). During this period, electrostatic interaction is weaker and van der Waals energy is stronger. Thus, for both of the best aptamers, a pi-pi bond between paraoxon and nucleotides can exist, though it seems that in aptamer $\mathrm{T} 17 \mathrm{C}-\mathrm{C} 18 \mathrm{~T}$ it is weaker and van der Waals interactions are more preferable.

In addition to the analysis of two most effective aptamers, it is interesting to analyze the behavior of other oligonucleotides in detail. For additional analysis, we have selected two the most ineffective aptamers (C18A and T20G, binding energies are $-13.4 \pm 16.0$ 
and $-18.1 \pm 11.0 \mathrm{kcal} / \mathrm{mol}$, respectively) and two aptamers with an average efficiency (T19C and C18G, binding energies are $-22.6 \pm 6.1$ and $-26.6 \pm 4.6 \mathrm{kcal} / \mathrm{mol}$, respectively). Fig. 8(c-f)shows the time dependence of van der Waals and electrostatic energies of the aptamers and paraoxon complexes.

After $3.8 \mathrm{~ns}$ of simulation of the complex of aptamer C18A and paraoxon, the distance between paraoxon and nucleotide A18 starts to increase (Fig. 3), and van der Waals energy also begins to increase from negative values towards zero (Fig. 8c). After 6 ns of simulation, the distance keeps increasing and electrostatic energy begins to increase too until paraoxon is completely detached from the aptamer and the values of interaction energies are equal to zero. Analysis of paraoxon behavior has shown that after $3.8 \mathrm{ns,}$ the phosphorous moiety of the ligand turns from the nucleotides into solution, and in the time interval from 5 to $6.5 \mathrm{~ns}$ the paraoxon molecule is bound to the aptamer only via pi-pi interaction with the aromatic ring of nucleotide T19. The complex of paraoxon with aptamer T20G behaves in a similar way (Fig. 3 and 8d). After 3.8 ns of simulation, the phosphorus moiety of the ligand turns into solution and the value of van der Waals energy increases. In the time interval between 4.5 to $6.5 \mathrm{~ns}$, paraoxon is bound with the aptamer only via pi-pi interaction with the aromatic rings of nucleotides T19 and G20, then paraoxon detaches from the aptamer.

It can be seen that in the complex of paraoxon with aptamer T19C the distance between the ligand and nucleotide C18 (Fig.3) and van der Waals energy (Fig. 8e) increase at the moments $2.2 \mathrm{~ns}$ and $8.5 \mathrm{~ns}$, then the values return to the initial ones. At these moments, as in the case of aptamers C18A and T20G, the phosphorous moiety of the ligand turns into solution, and paraoxon interacts with the aptamer only via pi-pi interaction with nucleotide T17, then the phosphorous moiety returns to its initial position. Similar 
behavior is observed for aptamer C18G. At time moments $4.2 \mathrm{~ns}$ and $8.5 \mathrm{~ns}$, both the distance between paraoxon and nucleotide C18 (Fig.3) and van der Waals energy (Fig. 8f) increase, then the values return to the initial ones. At these moments, the phosphorous moiety of the paraoxon turns into solution, and the ligand interacts with the aptamer via pi-pi interaction with nucleotides T19 and T20.

It is noteworthy that in the case of aptamers C18A, T20G and C18G, nucleotide T19 participates in pi-pi interaction with paraoxon. In the case of aptamer T19C, when nucleotide T19 is replaced by cytosine, it is nucleotide T17 that participates in this interaction. Thus, thymine is probably preferable to cytosine to form a pi-pi bond with paraoxon. Also it can be noted that pi-pi interaction is the last "anchor" for paraoxon before detaching from an aptamer molecule. A similar situation probably occurs in the opposite direction: before binding, a paraoxon molecule anchors to the aptamers via pipi interaction with one of the nucleotides of the binding site.

It is interesting to compare our observations with other experimental data. There are several complexes of aptamers with small molecules analyzed by nuclear magnetic resonance (NMR) in the Protein Data Bank (Berman et al., 2000). Unfortunately, there are no structures analogous to paraoxon. We have therefore selected the most similar structures for analysis: the complex of citrulline with an RNA aptamer (structure code 1kod, Yang et al., 1996); the complex of arginine with an RNA aptamer (structure code 1koc, Yang et al., 1996); the complex of two molecules of argininamide with an RNA aptamer (structure code 1nbk, Matsugami et al., 2003); and the complex of malachite green with an RNA aptamer (structure code 1q8n, Flinders et al., 2004). 
The structure of a citrulline molecule contains one $\mathrm{COO}^{-}$-group and one amide $\mathrm{C}(\mathrm{O}) \mathrm{NH}_{2}$-group. Analysis of the aptamer-citrulline complex has revealed that these groups do not form any specific bonds. The structure of an arginine molecule contains two $\mathrm{NH}_{2}$-groups and one $\mathrm{COO}^{-}$-group. One of the $\mathrm{NH}_{2}$ groups of the ligand may form a hydrogen bond with the aromatic nitrogen atom of one of the cytosines of the aptamer, but this is uncertain as some hydrogen atoms are missing in this structure. Furthermore, the $\mathrm{COO}^{-}$-group of arginine does not form any h-bonds since the distance to the other atoms is too great. The structure of an arginineamide molecule contains a $\mathrm{COO}^{-}$-group and a $\mathrm{NH}_{2}$-group. According to the geometry of an aptamer complex with two argininamides, $\mathrm{COO}^{-}$-groups of both ligands form hydrogen bonds with $\mathrm{NH}_{2}$-groups of cytosines. $\mathrm{NH}_{2}$-groups of both argininamides, according to the complex geometry, form hydrogen bonds with the aromatic nitrogen atoms of guanines. The structure of a malachite green molecule contains three aromatic rings. In its complex with an aptamer, two rings form two pi-pi bonds with aromatic rings of guanine and cytosine, according to their mutual conformation.

Based on the analysis of the NMR experimental data and our own results, we presume that stabilization of a ligand inside an aptamer binding site is not always determined by h-bonds, pi-pi or cation-pi interactions, even if an aptamer and a ligand have the groups that can form such interactions. Therefore, in our opinion, based on the currently available data, it is impossible to simplify the approach presented in this paper. We assume that it could be possible in future, when more structures of aptamers for small molecules are determined experimentally and by applying in silico tools. Statistical processing of these data could then help to develop a new, more rational search algorithm. 


\section{Conclusions}

Having applied rational in silico design, for the first time we have obtained aptamers that could bind paraoxon. We have shown that paraoxon binds to these aptamers more efficiently than to the peripheral anionic site of acetylcholinesterase. A similar approach could be used to search for aptamers for other OPs. Having analyzed the aptamerparaoxon complexes obtained, we conclude that h-bonds, pi-pi or cation-pi interactions can help anchoring a ligand by an aptamer, but stabilization of a ligand inside a binding site can be due to non-specific van der Waals interaction. Therefore, it is not possible currently to predict the structure of aptamers for OPs based on the chemical structure of a ligand.

\section{Acknowledgments}

These studies were carried out within the State assignment AAAA-A18-1180122901429 and supported in part by the Russian Foundation for Basic Research (project No. 18015-00304).

\section{Declaration of Interest}

The authors report no declarations of interest. 


\section{References}

Åqvist, J., Medina, C., Samuelsson, J.E., 1994. A new method for predicting binding affinity in computer-aided drug design. Protein Eng. 7, 385-391.

Ashrafuzzaman, M., Tseng, C.Y., Kapty, J., Mercer, J.R., Tuszynski. J.A., 2013.

Computationally designed DNA aptamer template with specific binding to phosphatidylserine. Nucleic Acid Ther. 23, 418-426.

Barril X., GelpíJ, J. L., López, J.M., Orozco, M., Luque, F. J., 2001. How accurate can molecular dynamics/linear response and Poisson-Boltzmann/solvent accessible surface calculations be for predicting relative binding affinities? Acetylcholinesterase huprine inhibitors as a test case. Theor. Chem. Acc. 106, 2-9.

Berendsen, H.J.C., Postma, J.P.M., di Nola, A., van Gunsteren, W.F., Haak, J.R., 1984. Molecular dynamics with coupling to an external bath . J. Chem. Phys. 81, 36843690.

Berendsen, H.J.C., Postma, J.P.M., van Gunsteren, W.F., Hermans, J., 1981. Interaction models for water in relation to protein hydration, in: Pullman, B. (Ed.), Intermolecular forces. Springer, Dordrecht, pp. 331-342.

Berendsen, H.J.C., van der Spoel, D., van Drunen, R., 1995. GROMACS: A messagepassing parallel molecular dynamics implementation. Comp. Phys. Comm. 91, 4356.

Berman, H.M., Westbrook, J., Feng, Z., Gilliland, G., Bhat, T.N., Weissig, H., Shindyalov, I.N., Bourne, P.E., 2000. The Protein Data Bank. Nucleic Acids Res. 28, $235-242$. 
BIOVIA, D. S., 2016. Discovery studio modeling environment. San Diego, DassaultSystemes, Release 2017.

Bouchard, P.R., Hutabarat, R.M., Thompson, K.M., 2010. Discovery and development of therapeutic aptamers. Annu. Rev. Pharmacol. Toxicol. 50, 237-257.

Bruno, J.G., Carrillo, M.P., Phillips, T., Vail, N.K., Hanson, D., 2008. Competitive FRET-aptamer-based detection of methylphosphonic acid, a common nerve agent metabolite. J. Fluoresc. 18, 867-876.

Bussi, G., Donadio, D., Parrinello, M., 2007. Canonical sampling through velocity rescaling. J ChemPhys 126, 014101.

Chang, A.L., McKeague, M., Liang, J.C., Smolke, C.D., 2014. Kinetic and equilibrium binding characterization of aptamers to small molecules using a label-free, sensitive, and scalable platform. Anal. Chem. 86, 3273-3278.

Cheung, J., Gary, E.N., Shiomi, K., Rosenberry, T.L., 2013. Structures of human acetylcholinesterase bound to dihydrotanshinone I and territrem B show peripheral site flexibility. ACS Med. Chem. Lett. 4, 1091-1096.

Conrad, R. C., Giver, L., Tian, Y., Ellington, A. D., 1996. In vitro selection of nucleic acid aptamers that bind proteins. Methods Enzymol. 267, 336 - 367.

Costa, L.G., 2006. Current issues in organophosphate toxicology. Clin. Chim. Acta. $366,1-13$.

Darden, T., York, D., Pedersen, L., 1993. Particle mesh Ewald: An N·log(N) method for Ewald sums in large systems. J. Chem. Phys. 3, 10089-10092. 
Dharmani, C., Jaga, K., 2005. Epidemiology of acute organophosphate poisoning in hospital emergency room patients. Rev. Environ. Health. 20, 215-232.

Fletcher, R., Reeves, C.M., 1964. Function minimization by conjugate gradients. Comput. J. 7, 148-154.

Flinders, J., DeFina, S.C., Brackett, D.M., Baugh, C., Wilson, C., Dieckmann, T., 2004. Recognition of planar and nonplanar ligands in the malachite green-RNA aptamer complex. Chembiochem. 5, 62-72.

Froimowitz, M., 1993. HyperChem: a software package for computational chemistry and molecular modeling. Biotechniques. 14, 1010-1013.

Genheden, S., 2011. MM/GBSA and LIE estimates of host-guest affinities: dependence on charges and solvation model. J. Comput. Aided Mol. Des. 25, 1085-1093.

Genheden, S., Ryde, U., 2015. The MM/PBSA and MM/GBSA methods to estimate ligand-binding affinities. Expert Opin. Drug Discov. 10, 449-461.

Goncharov, N. V., Nadeev, A. D., Jenkins, R. O., Avdonin, P. V., 2017. Markers and biomarkers of endothelium: when something is rotten in the state. Oxid. Med. Cell Longev. 2017, 9759735.

Hess, B., Bekker, H., Berendsen, H.J.C., Fraaije, J.G.E.M., 1997. LINCS: A linear constraint solver for molecular simulations. J. Comp. Chem. 8, 1463-1473.

Homeyer, N., Stoll, F., Hillisch, A., Gohlke, H., 2014. Binding free energy calculations for lead optimization: assessment of their accuracy in an industrial drug design context. J. Chem. Theory Comput. 10, 3331-3344. 
Hou, T., Guo, S., Xu, X., 2002. Predictions of binding of a diverse set of ligands to gelatinase-a by a combination of molecular dynamics and continuum solvent models. J. Phys. Chem. B. 106, 5527-5535.

Hsieh, P.C., Lin, H.T., Chen, W.Y., Tsai, J.J.P., Hu, W.P., 2017. The combination of computational and biosensing technologies for selecting aptamer against prostate specific antigen. Biomed. Res. Int. 5041683.

Hüttenhofer, A., Vogel, J., 2006. Experimental approaches to identify non-coding RNAs. Nucleic Acids Res. 34, 635-646.

Jensen, J.H., 2010. Molecular Modeling Basics. CRC Press, Boca-Raton.

Jorgensen, W.L., Chandrasekhar, J., Madura, J. D., Impey, R.W., Klein, M.L., 1983. Comparison of simple potential functions for simulating liquid water. J. Chem. Phys. 79, 926-935.

Kaur, S., Singh, S., Chahal, K.S., Prakash, A., 2014. Potential pharmacological strategies for the improved treatment of organophosphate-induced neurotoxicity. Can. J. Physiol. Pharmacol. 92, 893-911.

King, A.M., Aaron, C.K., 2015. Organophosphate and carbamate poisoning. Emerg. Med. Clin. North Am. 33, 133-151.

Ku, T.H., Zhang, T., Luo, H., Yen, T.M., Chen, P.W., Han, Y., Lo, Y.H., 2015. Nucleic acid aptamers: an emerging tool for biotechnology and biomedical sensing. Sensors (Basel). 15, 16281-16313. 
Kulbachinskiy, A.V., 2007. Methods for selection of aptamers to protein targets. Biochemistry (Mosc). 72, 1505-1518.

Kumari, R., Kumar, R., Open Source Drug Discovery Consortium, Lynn, A., 2014. g_mmpbsa - a GROMACS tool for high-throughput MM-PBSA calculations. J. Chem. Inf. Model.54, 1951-1962.

Lotti, M., Moretto, A., Capodicasa, E., Bertolazzi, M., Peraica, M., Scapellato, M.L., 1993. Interactions between neuropathy target esterase and its inhibitors on the development of polyneuropathy. Toxicol. Appl. Pharmacol. 122, 165-171.

Matsugami, A., Kobayashi, S., Ouhashi, K., Uesugi, S., Yamamoto, R., Taira, K., Nishikawa, S., Kumar, P.K., Katahira, M., 2003. Structural basis of the highly efficient trapping of the HIV Tat protein by an RNA aptamer. Structure. 11, 533-545.

Mikulskis, P., Genheden, S., Rydberg, P., Sandberg, L., Olsen, L., Ryde, U., 2012. Binding affinities in the SAMPL3 trypsin and host-guest blind tests estimated with the MM/PBSA and LIE methods. J. Comput. Aided. Mol. Des. 26, 527-541.

Morris, G.M., Goodsell, D.S., Halliday, R.S., Huey, R., Hart, W.E., Belew, R.K., Olson, A.J., 1998. Automated docking using a Lamarckian genetic algorithm and an empirical binding free energy function. J. Comput. Chem. 19, 1639-1662.

Perdih, A., Wolber, G., Solmajer, T., 2013. Molecular dynamics simulation and linear interaction energy study of D-Glu-based inhibitors of the MurD ligase. J. Comput. Aided. Mol. Des. 27, 723-738.

Peter, J.V., Jerobin, J., Nair, A., Bennett, A., Samuel, P., Chrispal, A., Abraham, O.C., Mathews, K.P., Fleming, J.J., Oommen, A., 2010. Clinical profile and outcome of 
patients hospitalized with dimethyl and diethyl organophosphate poisoning. Clin. Toxicol. (Phila). 48, 916-923.

Pitschmann, V., 2014. Overall View of Chemical and Biochemical Weapons. Toxins (Basel).6, 1761-1784.

Pope, C., Karanth, S., Liu, J., 2005. Pharmacology and toxicology of cholinesterase inhibitors: uses and misuses of a common mechanism of action. Environ. Toxicol. Pharmacol. 19, 433-446.

Radić, Z., Reiner, E., Taylor, P., 1991. Role of the peripheral anionic site on acetylcholinesterase: inhibition by substrates and coumarin derivatives. Mol. Pharmacol. 39, 98-104.

Radilov, A., Rembovskiy, V., Rybalchenko, I., Savelieva, E., Podolskaya, E., Babakov, V., Ermolaeva, E., Dulov, S., Kuznetsov, S., Mindukshev, I., Shpak, A., Krasnov, I., Khlebnikova, N., Jenkins, R., Goncharov, N, 2009. Russian VX, in: Gupta, R.C. (Ed.), Handbook of the Toxicology of Chemical Warfare Agents. Academic Press/Elsevier, Amsterdam, pp. 69-91.

Ray, D.E., 1998. Chronic effects of low level exposure to anticholinesterases--a mechanistic review. Toxicol Lett. 102-103, 527-533.

Rousseau, J.M., Rüttimann, M., Brinquin L., 2000. [Acute neurotoxic organophosphate poisoning: insecticides and chemical weapons]. Ann. Fr. Anesth. Reanim. 19, 588598.

Shcherbinin, D.S., Gnedenko, O.V., Khmeleva, S.A., Usanov, S.A., Gilep, A.A., Yantsevich, A.V., Shkel, T.V., Yushkevich, I.V., Radko, S.P., Ivanov A.S., 
Veselovsky, A.V., Archakov, A.I., 2015. Computer-aided design of aptamers for cytochrome p450. J. Struct. Biol. 191, 112-119.

Stoltenburg, R., Reinemann, C., Strehlitz, B., 2007. SELEX--a (r)evolutionary method to generate high-affinity nucleic acid ligands. Biomol. Eng. 24, 381-403.

Wang, L., Liu, X., Zhang, Q., Zhang, C., Liu, Y., Tu, K., Tu, J., 2012. Selection of DNA aptamers that bind to four organophosphorus pesticides. Biotechnol. Lett. 34, 869-874.

Williams, R.M., Maher, E., Sooter, L.J., 2014. In vitro selection of a single-stranded DNA molecular recognition element for the pesticide malathion. Comb. Chem. High Throughput Screen. 17, 694-702.

Yang, Y., Kochoyan, M., Burgstaller, P., Westhof, E., Famulok, M., 1996. Structural basis of ligand discrimination by two related RNA aptamers resolved by NMR spectroscopy. Science. 272, 1343-1347.

Zhang, C., Wang, L., Tu, Z., Sun, X., He, Q., Lei, Z., Xu, C., Liu, Y., Zhang, X., Yang, J., Liu, X., Xu, Y., 2014. Organophosphorus pesticides detection using broadspecific single-stranded DNA based fluorescence polarization aptamer assay. Biosensors and Bioelectronics. 55, 216-219.

Zhu, X., Lopes, P.E., Mackerell, A.D. Jr., 2012. Recent developments and applications of the CHARMM force fields. Wiley Interdiscip. Rev. Comput. Mol. Sci. 2, 167-185. 


\section{Caption to figures}

Fig. 1. 3D structure of aptamer SS24-S-35 (Zhang et al., 2014) obtained by molecular dynamic simulation.

Fig. 2.Possible binding sites of paraoxon inside the aptamer according to molecular docking.

Fig. 3. Time dependence of distance between the phosphorus atom of paraoxon and the phosphorus atom of a nucleotide at position 19 (as the approximate center of site-1) of the studied aptamers according to MD simulation. The values of the distances were taken every 100 ps during the simulations.

Fig. 4. Complex of paraoxon with site-1 of aptamer SS24-S-35 obtained by $10 \mathrm{~ns}$ molecular dynamics simulation.

Fig.5. Paraoxon molecule (presented in balls) bound in the peripheral anionic site (PAS) of acetylcholinesterase (AChE, presented as a white/grey surface). Amino acids of PAS Tyr72 and Asp74 are presented in sticks.

Fig. 6. The conformation of the complex of paraoxon (purple) with aptamer SS24-S-35 (grey) withthe lowest binding energy (Conf $\mathrm{best}_{\text {) }}$ ) according to MM-PBSA calculations and position of paraoxon (green) obtained by molecular docking into the aptamer in theConf best. $_{\text {. }}$

Fig. 7. The complexes of paraoxon with the site- 1 of aptamers T17C-C18T (A) and T17C (B) according to molecular dynamics data. 
Fig. 8. Time dependence of electrostatic (red) and van der Waals (blue) energy of interaction of paraoxon with aptamers T17C-C18T (A), T17C (B), C18A (C), T20G (D), T19C (E) and C18G (F) according to MM-PBSA calculations. 

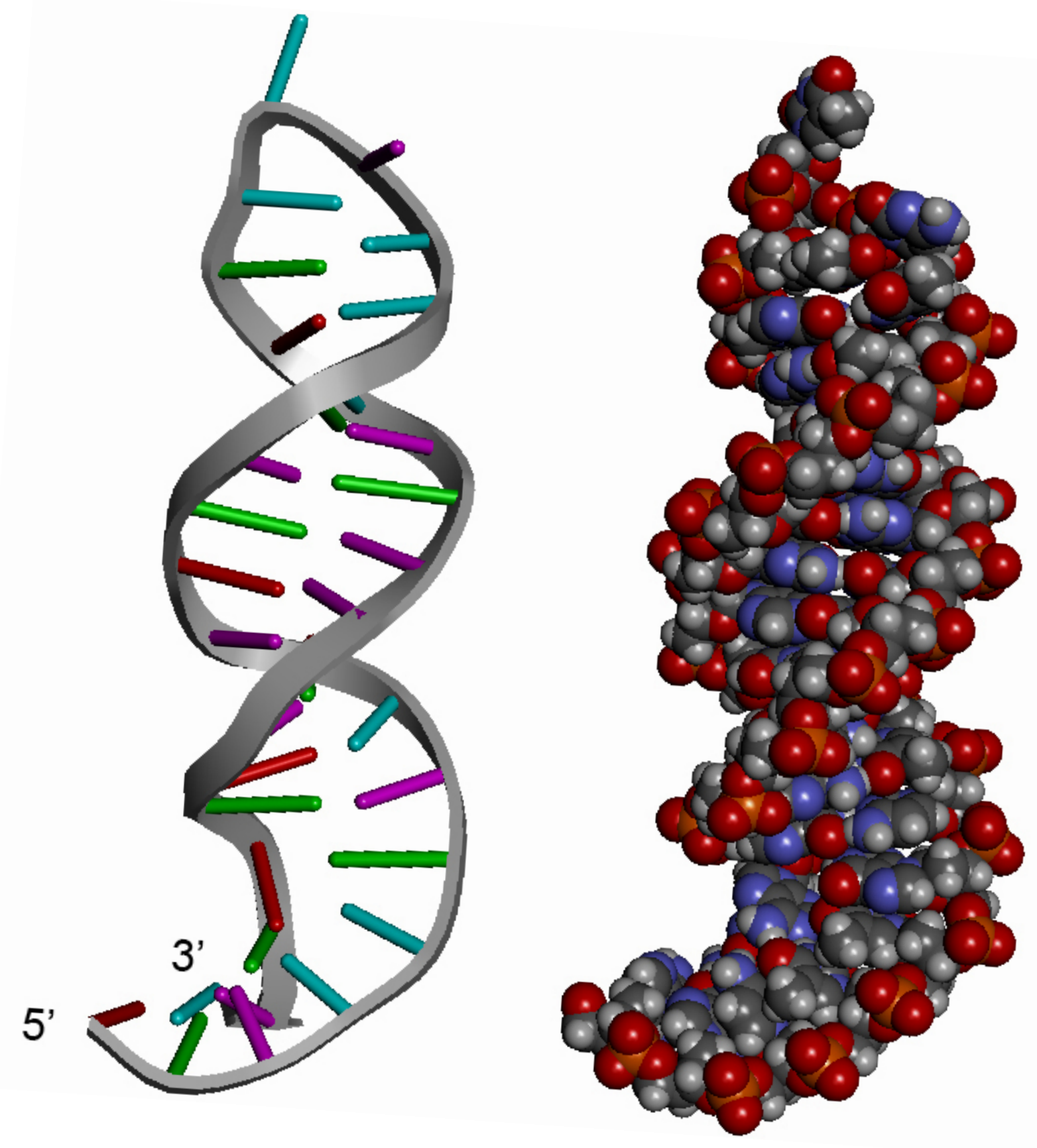


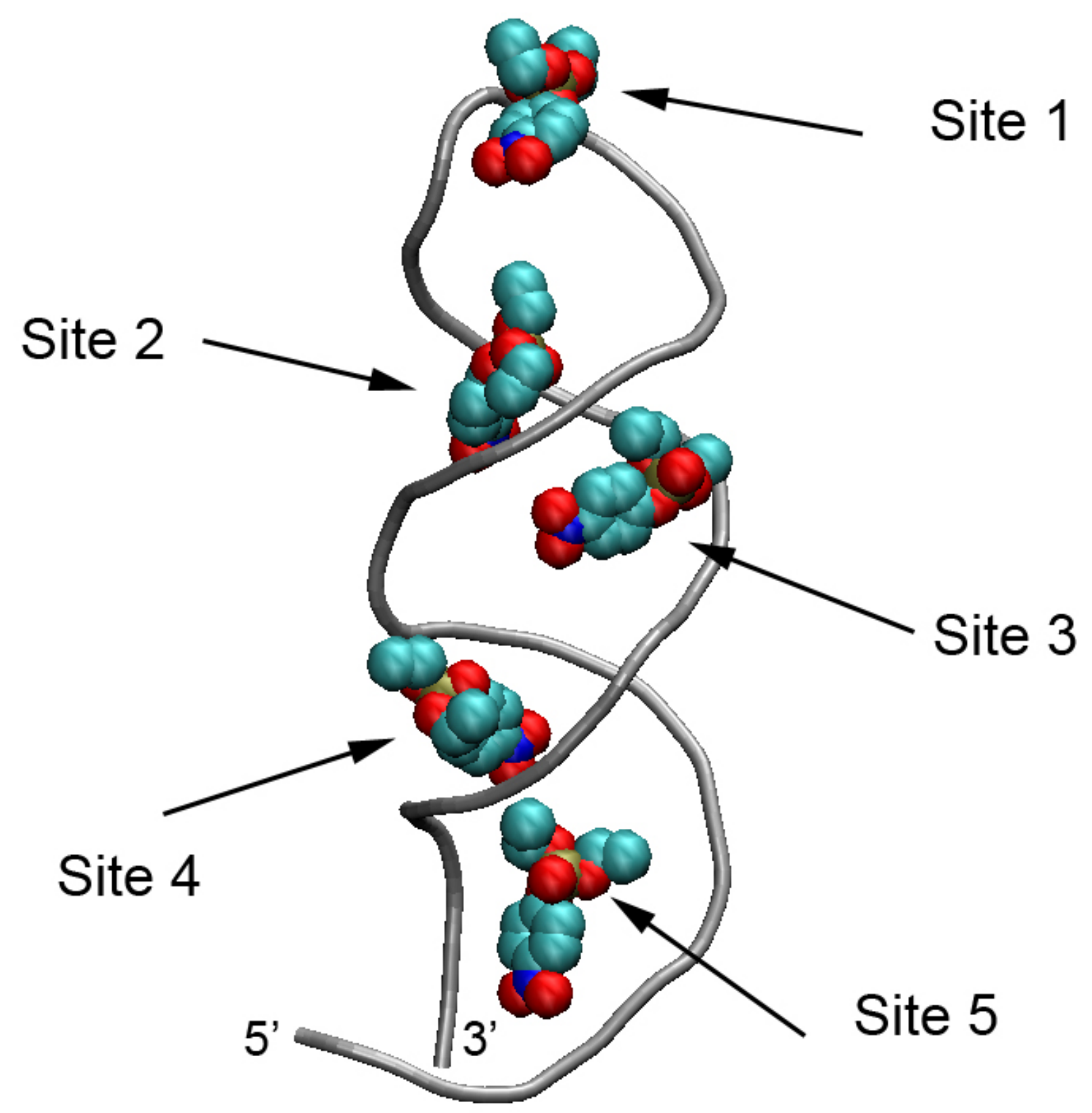




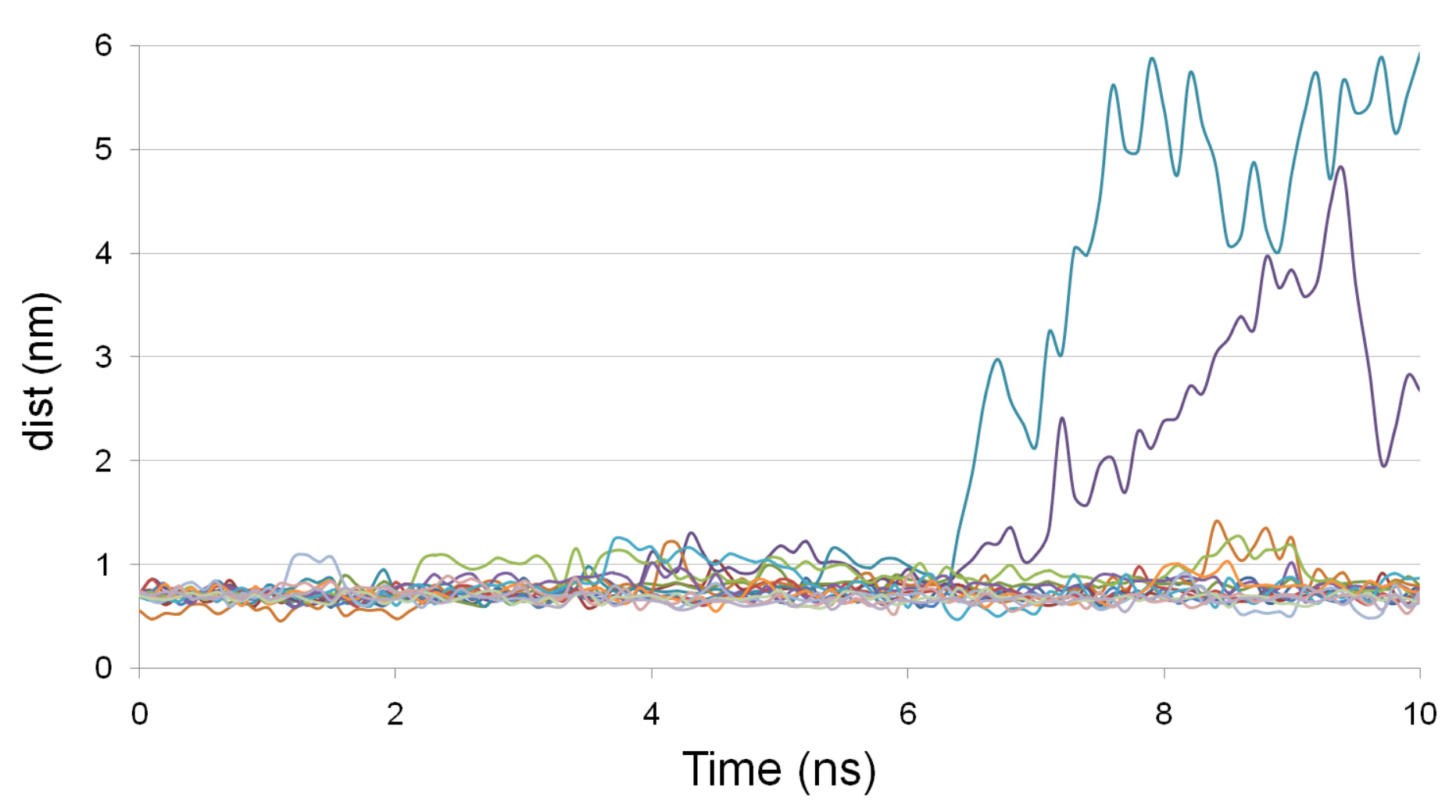

$-\mathrm{PAC}$
$-\mathrm{T} 20 \mathrm{~A}$
$-\mathrm{T} 20 \mathrm{C}$
$-\mathrm{T} 20 \mathrm{G}$
$-\mathrm{C} 18 \mathrm{~A}$
$-\mathrm{C} 18 \mathrm{G}$
$-\mathrm{C} 18 \mathrm{~T}$
$-\mathrm{T} 19 \mathrm{~A}$
$-\mathrm{T} 19 \mathrm{C}$
$-\mathrm{T} 19 \mathrm{G}$
$-\mathrm{T} 17 \mathrm{~A}$
$-\mathrm{T} 17 \mathrm{C}$
$-\mathrm{T} 17 \mathrm{G}$
$-\mathrm{T} 17 \mathrm{C}-\mathrm{C} 18 \mathrm{~T}$
$-\mathrm{T} 17 \mathrm{C}-\mathrm{T} 20 \mathrm{~A}$
$-\mathrm{T} 17 \mathrm{C}-\mathrm{C} 18 \mathrm{~T}-\mathrm{T} 20 \mathrm{~A}$




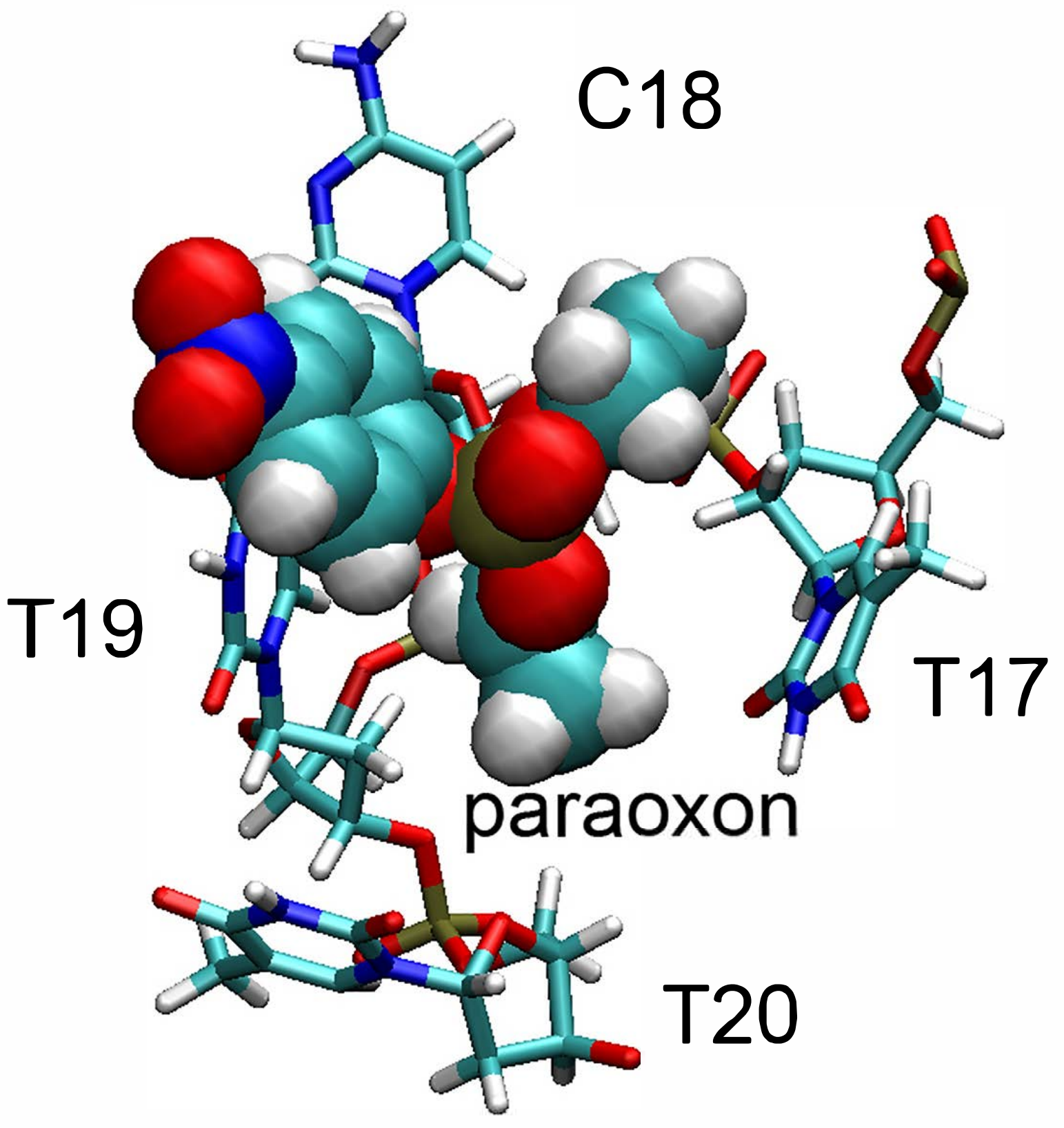




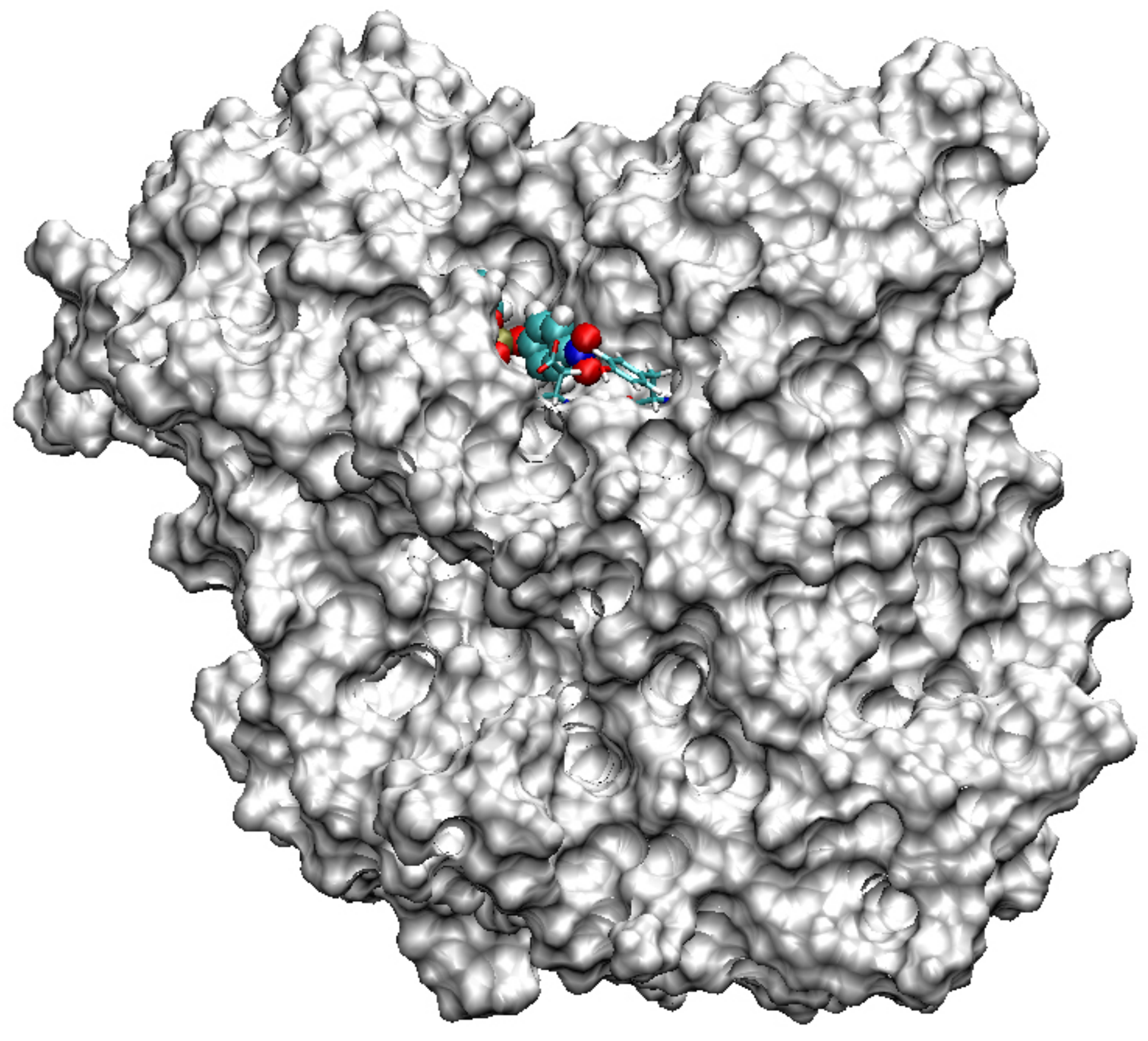




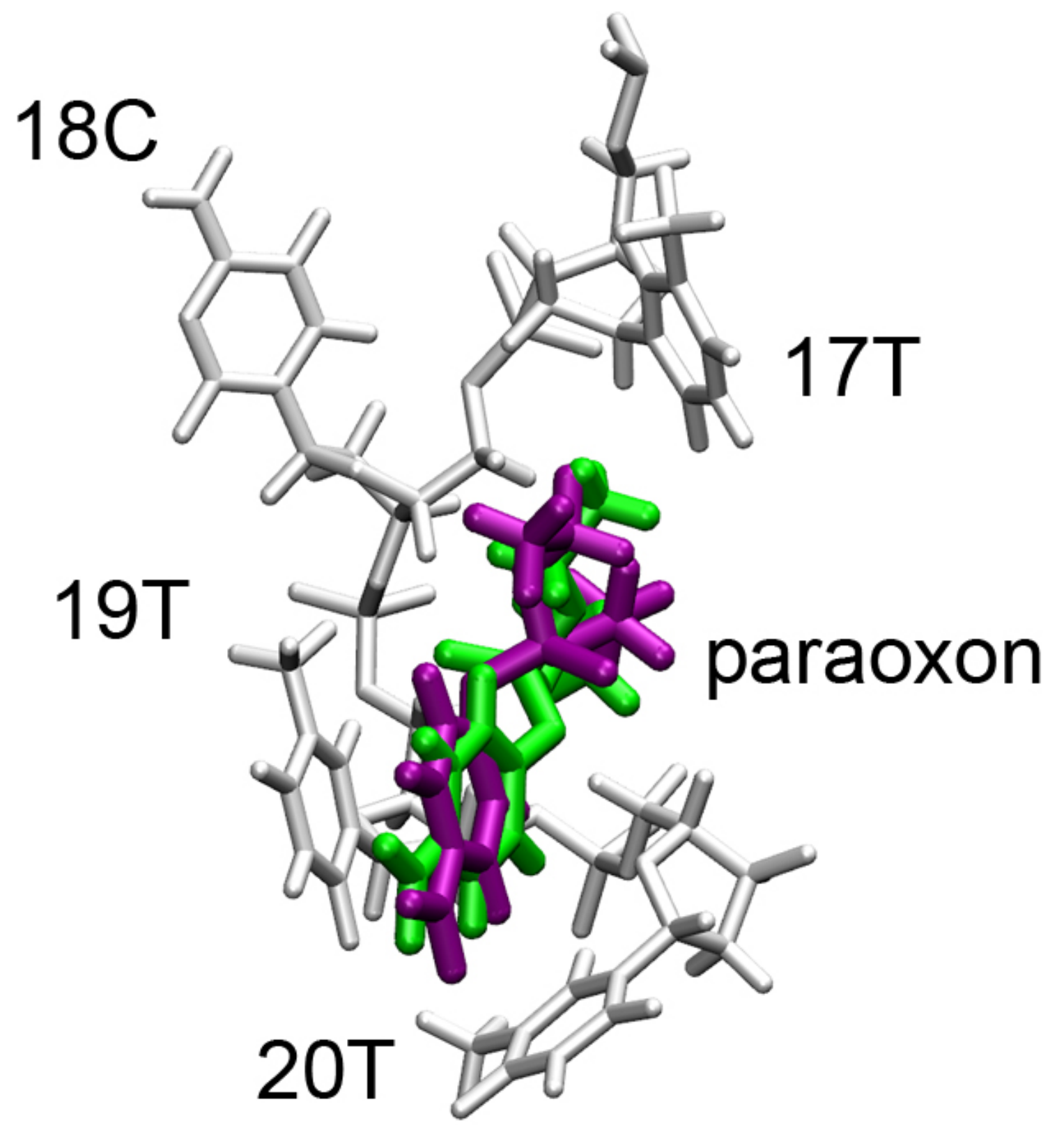


A

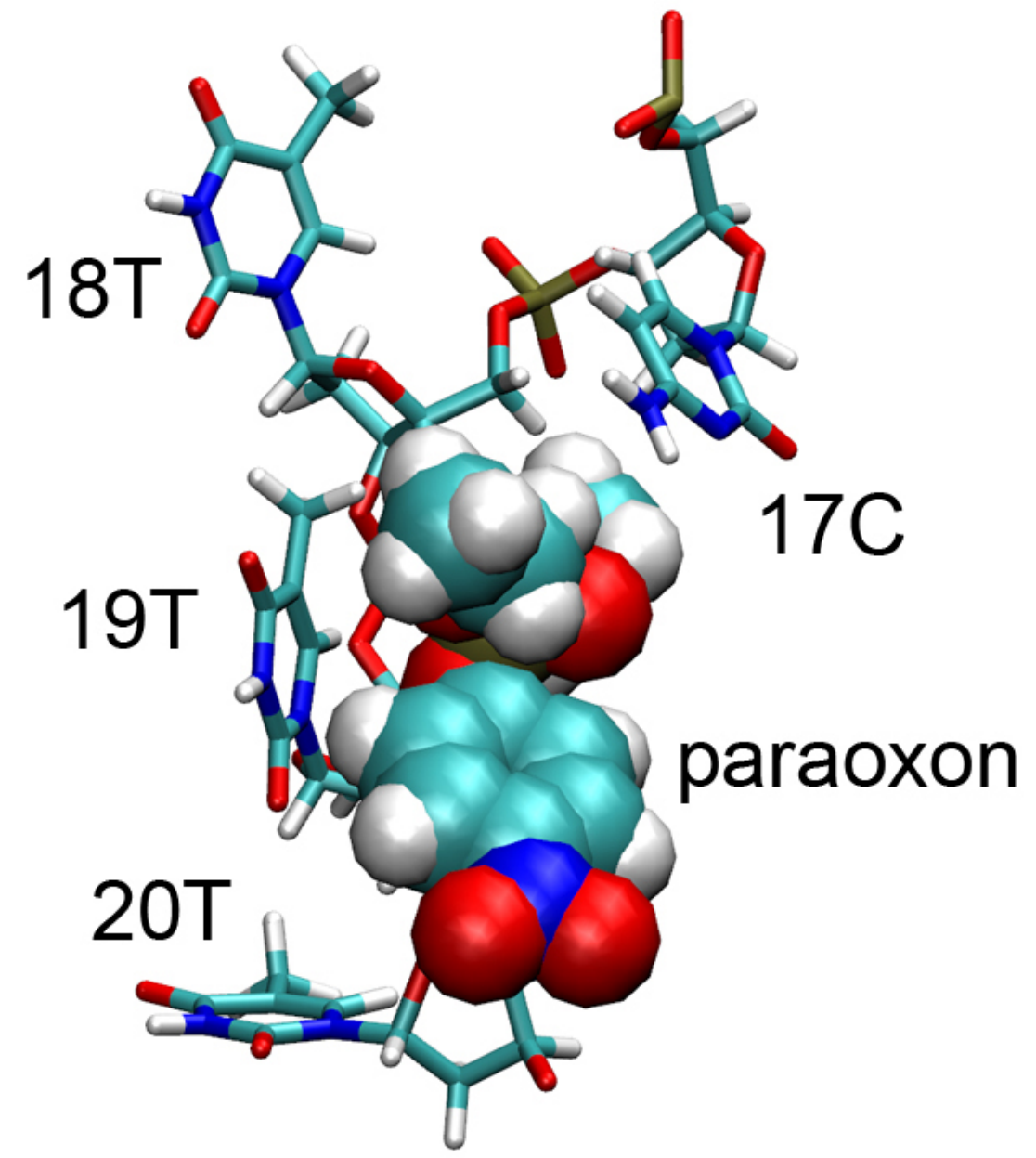

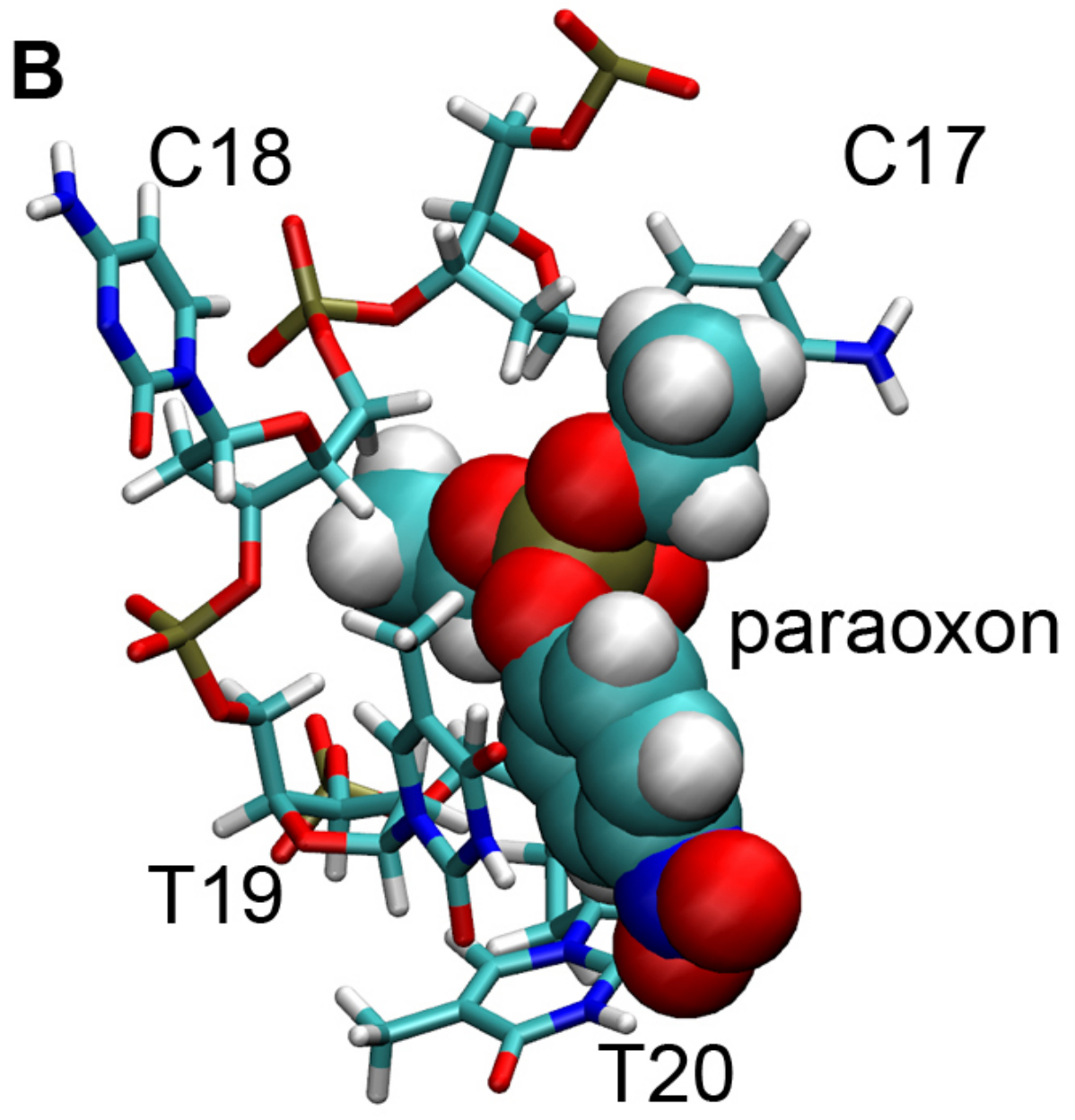



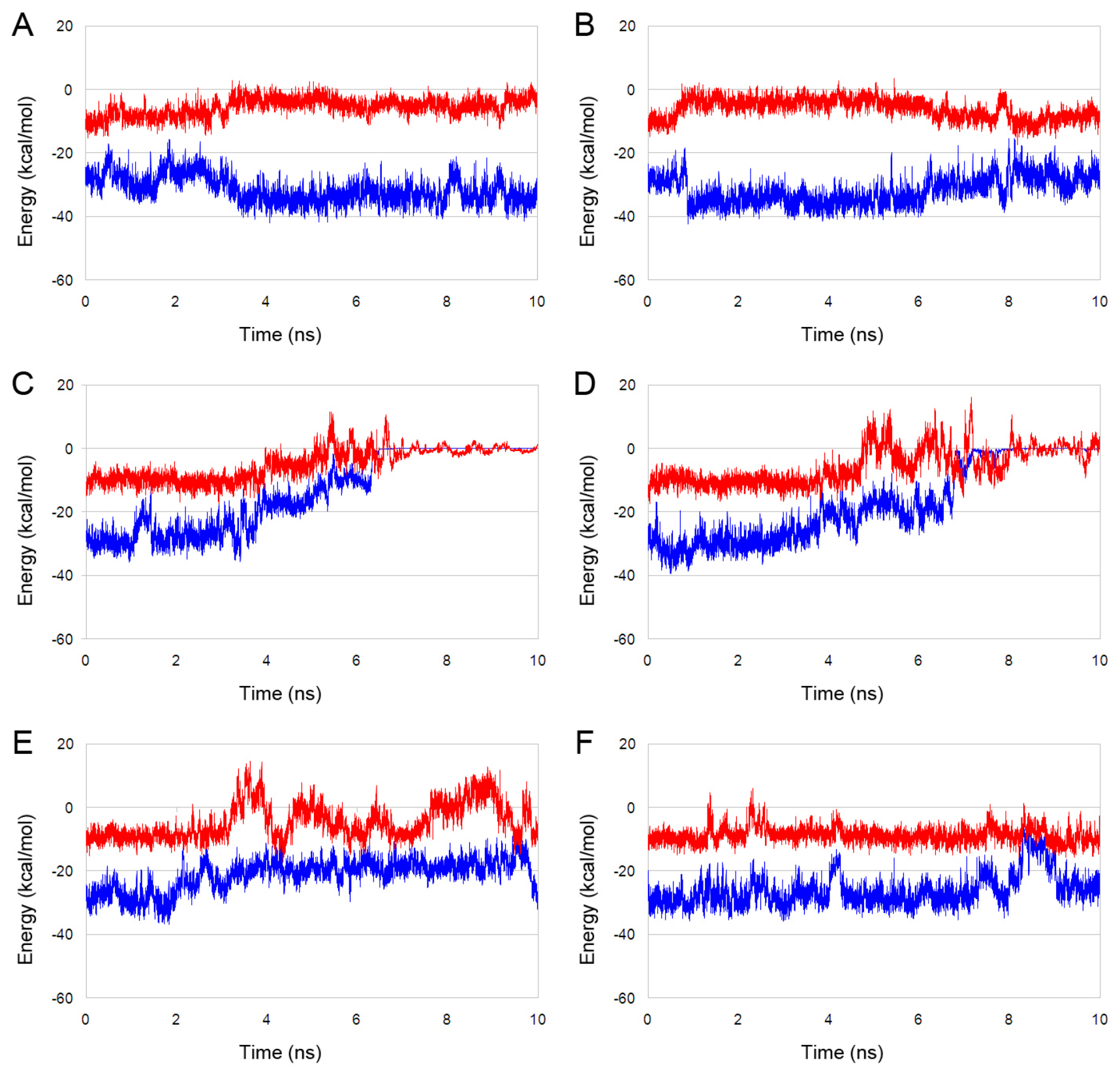
Table 1 Result of molecular docking of paraoxon into five possible binding sites of aptamer SS24-S-35 (aptamer obtained in Zhang et al. 2014).

\begin{tabular}{cccc}
\hline $\begin{array}{c}\text { Binding } \\
\text { site }\end{array}$ & $\begin{array}{c}\text { Nucleotides of the } \\
\text { binding site }\end{array}$ & $\begin{array}{c}\text { Intermolecular } \\
\text { energy, } \mathbf{k c a l} / \mathbf{m o l}\end{array}$ & $\begin{array}{c}\text { Estimated free energy } \\
\text { of binding, kcal/mol }\end{array}$ \\
\hline Site-1 & C18, T20 & -4.38 & -2.29 \\
Site-2 & C13, A15, A22, & -4.68 & -2.59 \\
Site-3 & G12, G25, C27 & -5.5 & -3.41 \\
Site-4 & C10, G31 & -6.25 & -4.16 \\
Site-5 & T5, G33 & -6.33 & -4.25 \\
\hline
\end{tabular}


Table 2 Contribution of the nucleotides of site-1 (T17, C18, T19, T20) to binding energy of the aptamer-paraoxon complex

\begin{tabular}{ccccc}
\hline Energy, kcal/mol & T17 & C18 & T19 & T20 \\
\hline MM kcal/mol & $-4.1 \pm 0.7$ & $-2.3 \pm 0.5$ & $-6.1 \pm 0.5$ & $-3.4 \pm 0.9$ \\
Polar kcal/mol & $0.1 \pm 0.6$ & $-0.3 \pm 0.7$ & $2.3 \pm 0.8$ & $1.3 \pm 0.9$ \\
SASA kcal/mol & $-0.3 \pm 0.1$ & $-0.1 \pm 0.1$ & $-0.4 \pm 0.1$ & $-0.3 \pm 0.1$ \\
Total kcal/mol & $-4.2 \pm 1.4$ & $-2.7 \pm 1.2$ & $-4.2 \pm 1.5$ & $-2.4 \pm 1.8$ \\
\hline
\end{tabular}

$\mathrm{MM}$ - potential energy in vacuum, polar - polar solvation energy, SASA - non-polar solvation energy, $\mathrm{T}$ - thymine, $\mathrm{C}$ - cytosine. 
Table 3 Free binding energies of the initial paraoxon-aptamer- complex (PAC) and of its mutants.

Initial paraoxon-aptamer complex

Free binding energy, kcal/mol
$\operatorname{PAC}\left(\mathrm{T}^{17} \mathrm{C}^{18} \mathrm{~T}^{19} \mathrm{~T}^{20}\right)$
$-31.0 \pm 3.8$

Mutation at position 20

Free binding

Aptamer

energy, $\mathrm{kcal} / \mathrm{mol}$
Mutation at position 18

Free binding

Aptamer

energy, $\mathrm{kcal} / \mathrm{mol}$

\begin{tabular}{cccc}
\hline T20A & $-30.7 \pm 3.9$ & $\mathrm{C} 18 \mathrm{~A}$ & $-13.4 \pm 16.0$ \\
$\mathrm{~T} 20 \mathrm{C}$ & $-27.3 \pm 4.0$ & $\mathrm{C} 18 \mathrm{G}$ & $-26.6 \pm 4.6$ \\
$\mathrm{~T} 20 \mathrm{G}$ & $-18.1 \pm 11.0$ & $\mathrm{C} 18 \mathrm{~T}$ & $-30.6 \pm 3.6$
\end{tabular}

Mutation at position 19

Mutation at position 17

Free binding

Aptamer

energy, $\mathrm{kcal} / \mathrm{mol}$

Aptamer

Free binding

\begin{tabular}{cccc} 
Aptamer & energy, kcal/mol & Aptamer & energy, kcal/mol \\
& $-25.5 \pm 9.9$ & T17A & $-27.5 \pm 4.9$ \\
T19A & $-22.6 \pm 6.1$ & T17C & $-32.3 \pm 3.0$ \\
T19C & $-29.4 \pm 5.0$ & T17G & $-31.3 \pm 3.7$ \\
\hline
\end{tabular}

Free biding energies were obtained by molecular docking (MD) 